\title{
Functional genetic characterization by CRISPR-Cas9 of two enhancers of FOXP2 in a child with speech and language impairment
}

Torres-Ruiz, R. ${ }^{1}$, Benítez-Burraco, A. ${ }^{2,3}$, Martínez-Lage, M. ${ }^{1}$, Rodríguez-Perales, S. ${ }^{1, *}$, and García-Bellido, $\mathrm{P}^{3,4, *}$

1. Molecular Cytogenetics Group. Centro Nacional Investigaciones Oncológicas (CNIO), Madrid, Spain.

2. Department of Philology, University of Huelva, Huelva, Spain.

3. Faculty of Modern Languages, University of Oxford, Oxford, United Kingdom.

4. Faculty of Linguistics, Philology and Phonetics, University of Oxford, Oxford, United Kingdom.

* Corresponding authors: Sandra Rodriguez-Perales: srodriguezp@ cnio.es; Phone: (+34) 912 246 900; Fax:

(+34) 912246 911. Paloma Garcia-Bellido: paloma.garcia-bellido@mod-langs.ox.ac.uk

Phone (+44) 1865 27049; Fax: (+44) 1865270757.

\section{ABSTRACT}

Mutations in the coding region of FOXP2 are known to cause speech and language impairment. Microdeletions involving the region downstream the gene have been also associated to speech and cognitive deficits. We recently described a girl harbouring a complex chromosomal rearrangement with one breakpoint downstream the gene that might affect her speech and cognitive abilities via physical separation of distant regulatory DNA elements. In this study, we have used highly efficient targeted chromosomal deletions induced by the CRISPR/Cas9 genome editing tool to demonstrate the functionality of two enhancers, FOXP2-E proximal $^{\text {and FOXP2-E }}{ }^{\text {distal }}$, located in the intergenic region between FOXP2 and its adjacent MDFIC gene. Deletion of any of these two functional enhancers in the neuroblastomic cell line SK-N-MC downregulates $F O X P 2$ and decreases FOXP2 protein levels, conversely it upregulates $M D F I C$ and increases MDFIC protein levels. This suggests that both regulatory elements may be shared between FOXP2 and MDFIC. We expect these findings contribute to a deeper understanding of how FOXP2 and MDFIC are regulated to pace neuronal development supporting speech and language.

\section{KEYWORDS}

FOXP2, MDFIC, speech and language impairment, Spanish, CRISP-genome editing, functional enhancer

\section{ACKNOWLEDGMENTS}


This project was supported by funds from The University of Oxford John Fell OUP Research Grant [121/435] awarded to its Principal Investigator Paloma Garcia-Bellido. This study was supported in part by funds from the Spanish National Research and Development Plan, Instituto de Salud Carlos III, and FEDER (FIS project no. PI14/01884 to Sandra Rodriguez-Perales), and in part by funds from the Spanish Ministry of Economy and Competitiveness (grant numbers FFI2014-61888-EXP and FFI-2013-43823-P to Antonio Benítez-Burraco). The funders had no role in the study design, data collection and analysis, decision to publish, or preparation of the manuscript. We thank Daniela Moralli, Dianne Newbury and Sonja C. Vernes for their helpful comments.

\section{CONFLICT OF INTEREST}

The authors declare that they have no conflict of interest.

\section{ETHICS}

Ethics approval for this research was granted by the University of Oxford [MSD-IDREC-C1-2012-95, SSD/CUREC2/09-23].

\section{AUTHORS CONTRIBUTION}

P G-B conceived the project, participated in the design and coordination of experiments, analysed results, and wrote the paper.

A B-B analysed results and wrote the paper.

$\mathrm{R}$ T-R did the experiments, analysed results, and wrote the paper.

M M-L did the experiments.

S R-P participated in the design, coordinated experiments, analysed results, and wrote the paper.

All authors read and approved the final manuscript 


\section{INTRODUCTION}

Mutations in the gene FOXP2, encoding a transcription factor, are known to cause speech and language impairment (Vargha-Khadem et al. 2005; Zhao et al. 2010). Polymorphisms of the gene have been also associated to schizophrenia (Tolosa et al. 2010) and frontotemporal lobar degeneration (Padovani et al. 2010). FOXP2 has been hypothesised to regulate the development and function of brain areas involved in human language processing (Lai et al., 2003; Fisher and Scharff 2009), because of its known role in neurogenesis, neuron differentiation and migration patterns in the developing telencephalon in mice (Tsui et al. 2013; Chiu et al. 2014; Garcia-Calero et al. 2016). Pathogenic mutations in humans have proven to impair auditory-motor association learning in mice (Kurt et al. 2012). Nonetheless, the exact role of FOXP2 in normal development is unknown. Common variants of the gene do not contribute appreciably to individual differences in language development (Mueller et al. 2016), nor in brain structure (Hoogman et al. 2014), although a FOXP2 polymorphism has been associated with enhanced procedural learning of nonnative speech sound categories (Chandrasekaran et al. 2015). Less is known about how the expression of the gene is modulated. The promoter of FOXP2 contains four transcription start sites (Schroeder and Myers 2008), with multiple alternative splicing sites (Bruce and Margolis 2002). FOXP2 also contains six ultraconserved regions in its introns (Bejerano et al. 2004, Schroeder and Myers 2008), as well as six predicted enhancers for Lef1 (Hallikas et al 2006). Lef1 is a transcription factor that drives expression of the Foxp2 gene in the central nervous system during zebrafish embryogenesis (Bonkowsky et al. 2008). Interestingly, several microRNAS bind the 3'UTR of the gene and regulate the expression of $F O X P 2$ (Clovis et al. 2012; Shi et al. 2013; Fu et al. 2014a; Cuiffo et al. 2014).

Apart from gene mutations, microdeletions involving FOXP2 and/or MDFIC, the adjacent gene downstream $F O X P 2$, and the region between these two genes have been found in subjects with speech delay and cognitive impairment (DECIPHER patients 262086, 292652, and 301696). We have recently reported on a young female harbouring a genomic complex rearrangement involving chromosomes 7 and 11 , who presents with severe expressive and receptive speech and language impairment in both Castilian Spanish and Valencian (Moralli et al. 2015). Although the FOXP2 coding region is intact, the breakpoint in 7q31.1 is located $205.5 \mathrm{~kb}$ downstream the 3' end of FOXP2 and $22.8 \mathrm{~kb}$ upstream the 5 ' region of MDFIC. Becker et al. (2015) found and characterized a functional enhancer located $2.5 \mathrm{~kb}$ downstream the breakpoint and hypothesized that rearrangement of this enhancer by chromosomal translocation may contribute to the 
observed language phenotype by disturbing FOXP2 gene expression. In our proband this element was hypothesized to be in derivative chromosome $7 \mathrm{q}$ separated from FOXP2. While FOXP2 was shown to have been rearranged to derivative chromosome $11 \mathrm{p}, M D F I C$ had been located in derivative chromosome 7q, based on the FISH results from the RP11-243D16 BAC (Moralli et al 2015). A more robust approach, aimed at looking for changes in the expression levels of the gene seems desirable in order to know if this enhancer regulates $F O X P 2$ expression.

The development of nuclease mediated genome editing tools, specially, of those based on clustering regularly interspaced short palindromic repeats (CRISPR) (Sakuma and Woltjen 2014; Torres-Ruiz and Rodriguez-Perales 2016), has emerged as a highly efficient way of inducing targeted chromosomal deletions and an accurate method to validate the functionality of enhancers (Cong et al. 2013; Mali et al. 2013). Here we report a detailed study of the intergenic region between the FOXP2 and MDFIC genes. We have found that this region contains, apart from the enhancer reported in Becker et al. (2015), a second functional enhancer, FOXP2-E proximal. We performed targeted deletions of each regulatory element by CRISPR-Cas9 and found that both affect the expression levels of FOXP2 and MDFIC in an opposite manner, increasing FOXP2 and reducing MDFIC mRNA and protein levels. We hypothesise therefore that the breakpoint in this case would cause FOXP2 to be anomalously downregulated by the separation of FOXP2-distal from FOXP2, while MDFIC to be anomalously upregulated by the separation of FOXP2_proximal from MDFIC. These changes in the expression levels of these two genes may account for the observed language deficits in this case. We expect these findings contribute to a better understanding of how FOXP2 is regulated.

\section{MATERIALS AND METHODS}

\section{Cell culture and electroporation}

Cells of the human non neuronal cell-line HEK293A (CRL-1573, ATCC, USA) and the neuroblastomic cell-line SK-N-MC (HTB-10, ATCC, USA) were maintained under standard conditions in Dulbecco's modified Eagle's medium (DMEM) (Lonza), supplemented with $10 \%$ foetal bovine serum (FBS) (Life Technologies), 1\% GlutaMAX (Life Technologies), and 10mg/ml penicillin/streptomycin (Life Technologies). The neuroblstomic cell-line SK-SY-5Y (CRL-2266, ATCC, USA) was cultured in a 1:1 mixture of Dulbecco's modified Eagle's medium (DMEM) (Lonza) and F12 medium (Lonza) supplemented 
with 10\% FBS (Life Technologies), $1 \%$ GlutaMAX (Life Technologies), and 10mg/ml penicillin/streptomycin (Life Technologies). Cells were cultured at $37^{\circ} \mathrm{C}$ in a humidified incubator in a $5 \%$ $\mathrm{CO}_{2}+20 \% \mathrm{O}_{2}$ atmosphere.

For electroporation, we used the Neon Transfection System (Life Technologies). The manufacturer's protocols for HEK293A, SK-N-MC and SH-SY5Y cells were modified as follows. The three cell types were electroporated at $80 \%$ confluence. Cells were trypsinized and resuspended in $\mathrm{R}$ solution (Life Technologies). For SK-N-MC and SH-SY5Y, 10- $\mu$ l tips were used to electroporate $2.5 \times 10^{6}$ cells with a single 50-ms pulse of $900 \mathrm{~V}$. For HEK293A cells, $4 \times 10^{5}$ cells were electroporated with $10-\mu 1$ tips using three 10-ms pulses of $1245 \mathrm{~V}$. After electroporation, cells were seeded in a 24 -well plate containing prewarmed medium. When required, cells were sorted $72 \mathrm{~h}$ post-transfection.

\section{Construction of Double-Guide Cas9-Encoding Plasmids}

The parental pLV-U6 ${ }^{\# 1} \mathrm{H} 1^{\# 2}$-C9G vector has been described elsewhere (Torres et al. 2014a). Two gBlocks

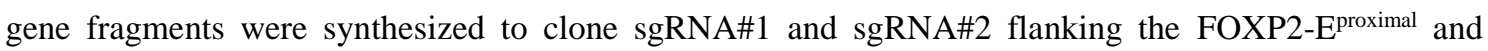

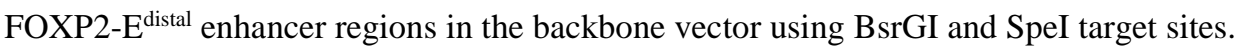

\section{Flow Cytometry and Cell Sorting}

72 hours after electroporation, cells were trypsinized and washed with DPBS twice, counted, and resuspended in an appropriate volume of sorting buffer (PBS containing 1\% FBS and antibiotics) for flow cytometry analysis. Immediately before cell sorting, samples were filtered through a $70-\mu \mathrm{m}$ filter to remove any clumps or aggregates. Cell sorting was carried out in a Synergy 2L instrument (Sony Biotechnology Inc.); flow cytometry was performed in a BD LSR Fortessa analyzer (BD Biosciences). Cells were sorted and seeded individually per well in a 96 well-plate.

\section{Genomic DNA Extraction and PCR Analysis}

Genomic DNA was extracted using standard procedures (Torres et al. 2014b). Briefly, 5-10×106 cells were either trypsinized or scraped, washed in PBS, pelleted, and lysed in $100 \mathrm{mM} \mathrm{NaCl}$, Tris (pH 8.0) 50mM, EDTA $100 \mathrm{mM}$, and $1 \%$ SDS. After overnight digestion at $56^{\circ} \mathrm{C}$ with $0.5 \mathrm{mg} / \mathrm{ml}$ of proteinase $\mathrm{K}$ (Roche Diagnostics), the DNA was cleaned by precipitation with saturated $\mathrm{NaCl}$, and the clear supernatant was 
precipitated with isopropanol, washed with $70 \%$ ethanol, air-dried, and resuspended overnight at room temperature in 1xTE buffer. Serial DNA dilutions were quantified with a NanoDrop ND 1000 Spectrophotometer (NanoDrop Technologies).

Standard PCR was performed in a Veriti 96-well Thermal Cycler (Applied Biosystems) under the following conditions: template denaturation at $95^{\circ} \mathrm{C}$ for $1 \mathrm{~min}$ followed by 30 cycles of denaturation at $94^{\circ} \mathrm{C}$ for $30 \mathrm{~s}$, annealing at $62.5^{\circ} \mathrm{C}$ for $30 \mathrm{~s}$, extension at $72^{\circ} \mathrm{C}$ for $60 \mathrm{~s}$, and a final extension of $5 \mathrm{~min}$ at $72^{\circ} \mathrm{C}$. Primers used are listed in Supplementary Table 1.

\section{$\underline{\text { RNA extraction and PCR }}$}

Total RNA was extracted from tissues and cell cultures using Trizol (Sigma-Aldrich), followed by treatment with RNase-free DNAse (Roche Applied Science). cDNA was synthesized from 500 ng of total RNA using the Superscript III First Strand cDNA Synthesis Kit (Life Technologies). Specific mRNAs were quantified by qRT-PCR using an ABI Prism 7900 HT Detection System (Applied Biosystems) and TaqMan detection. PCR was performed in 96-well plate microtest plates with TaqMan master mix (Thermo Fisher) for 40 cycles. In all experiments, mRNA amounts were normalized to the total amount of cDNA by using amplification signals for hGUSB. Each sample was determined in triplicate, and at least three independent samples were analysed for each experimental condition.

\section{Western Blot}

Proteins were extracted by standard procedures as previously described (Rodriguez-Perales et al. 2015) in the presence of Complete Protease Inhibitor Cocktail Tables (Roche Applied Science). Proteins were transferred to PVDF using TransFi (Invitrogen; Life Technologies), and membranes were probed for FOXP2 or MDFIC with monoclonal mouse anti-human FOXP2 or MDFIC antibodies (1/1000 or 1/500; BD Pharmigen) or for GAPDH (AbCam), with antibodies diluted 1/2500 in PBS/0.1\% Tween-20 (PBS-T). Secondary antibodies were HRP-conjugated with goat anti-mouse IgG (1/1000) and goat anti-Rabbit (1/500; Dako, Barcelona, Spain), and blots were developed with ECL (GE Healthcare).

\section{$\underline{\text { Statistical Analysis }}$}

Data from three or more independent experiments were analysed by two-tailed unpaired t-test. NS, nonsignificant; $* \mathrm{p}<0.05 ; * * \mathrm{p}<0.01 ; * * * \mathrm{p}<0.001$; and $* * * * \mathrm{p}<0.0001$. 


\section{RESULTS}

\section{In silico search of enhancer regions}

We first hypothesised that the breakpoint in $7 \mathrm{q} 31.1(114,888,284 \mathrm{hg} 38)$ affected the expression of FOXP2 by physically separating some cis-acting distant element with an enhancer role. Accordingly, we searched in silico for putative enhancers in the intergenic region between FOXP2 and MDIFC looking for the following hallmarks: DNase I hypersensitive sites, presence of histones with specific post-translational modifications (specifically histone $\mathrm{H} 3$, lysine 4 monomethylation (H3K4me1) and $\mathrm{H} 3$ lysine 27 acetylation (H3K27ac)), and ChIP-seq data provided by ENCODE of regions recruiting co-activators and co-repressors as revealed by chromatin immunoprecipitation followed by deep sequencing. We found two putative enhancers located at $120 \mathrm{~kb}$ and $203.5 \mathrm{~kb}$ downstream the stop codon of FOXP2, respectively (Figure $1 \mathrm{~A}$ and 1B). These putative enhancers (referred as FOXP2-E Eroximal $^{\text {and FOXP2-E }}$ distal $^{\text {) }}$ span 6264bp (chr7:114,817,431-114,823,694 hg38) and 2300bp (chr7:114,900,989-114,903,302 hg38 equivalent to $114,541,370-114,542,201 \mathrm{hg} 19)$, respectively. FOXP2-E $\mathrm{E}^{\mathrm{distal}}$ is the one previously validated by luciferase

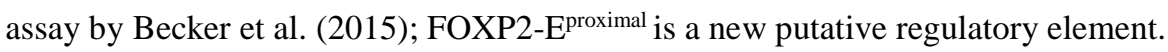

\section{CRISPR deletion of FOXP2-E proximal $^{\text {and FOXP2-E }}{ }^{\text {distal }}$}

We then tested in vitro the functionality of FOXP2-E $E^{\text {proximal }}$ and FOXP2-E $E^{\text {distal }}$. Since both putative enhancers are located in an intergenic region, we aimed at characterizing that both of them are functional with respect to FOXP2 or/and MDFIC. We relied on a CRISPR genome editing approach to delete the entire predicted sequence of each enhancer. Accordingly, we designed two couples of sgRNAs targeting

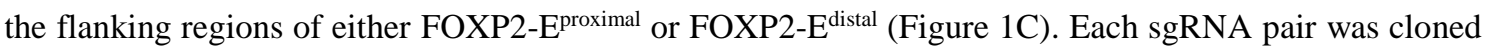
in the pLV-U6 $6^{\# 1} \mathrm{H} 1^{\# 2}-\mathrm{C} 9 \mathrm{G}$ (Torres et al. 2014b) in order to couple the expression of the sgRNAs to the expression of Cas9 and a GFP reporter. Subsequently, we tested if the sgRNAs were able to induce the expected deletions. HEK293A cells were nucleofected with 2 ug of pLV-U6 ${ }^{\# 1} \mathrm{H}^{\# 2}$-C9G plasmid targeting either FOXP2-E proximal $^{\text {or FOXP2-E }}{ }^{\text {distal }}$. After $72 \mathrm{~h}$, the DNA was isolated and analyzed. After designing PCR oligos that span the deleted regions (Supplementary Table 1), PCR assays were performed. They revealed efficient targeted deletions of the $6.2 \mathrm{~kb}$ or the $2.3 \mathrm{~kb}$ regions containing the entire sequence of FOXP2-E proximal and FOXP2-E $^{\text {distal }}$, respectively (data not shown). 
A

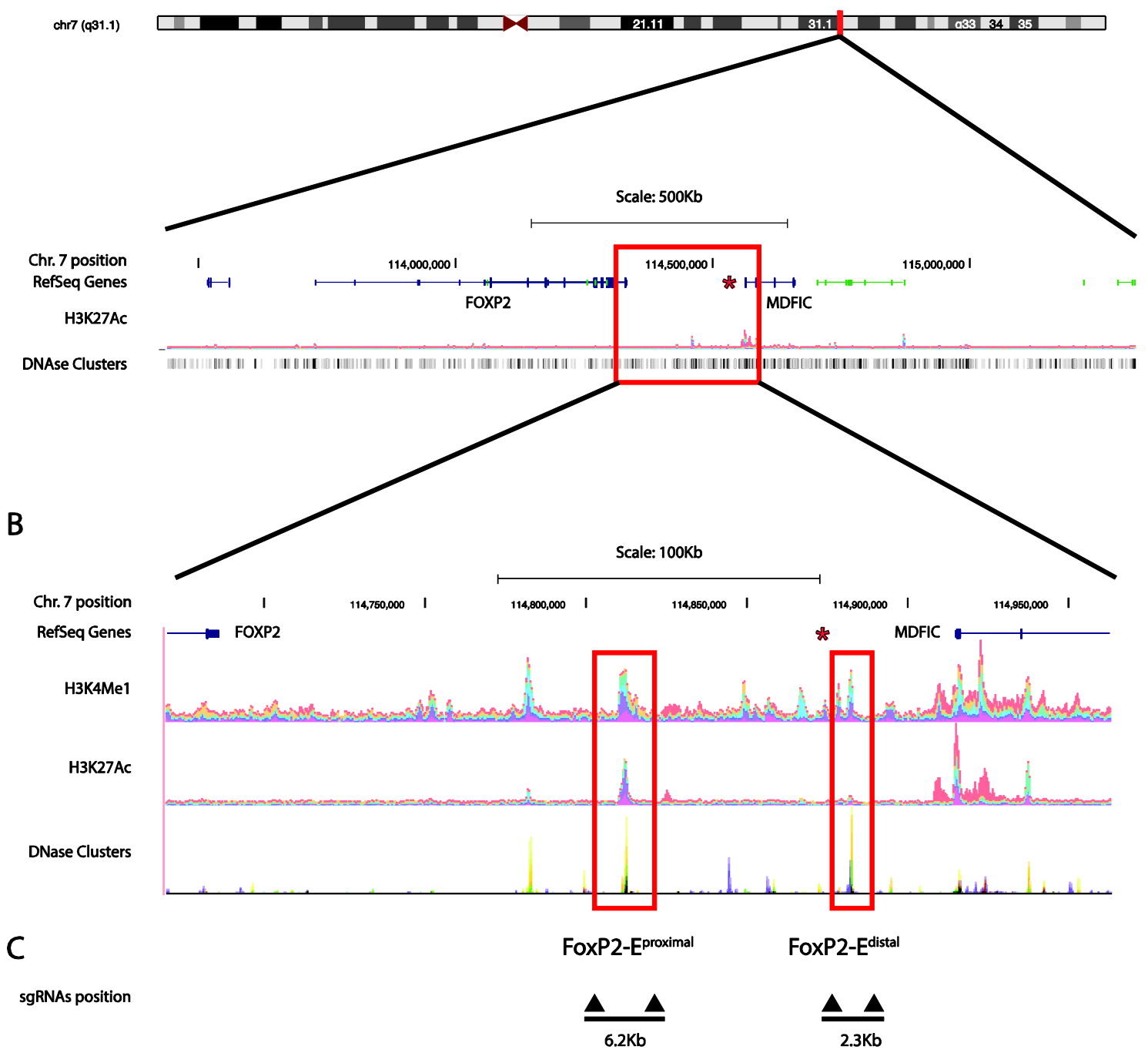

Figure 1. Identification of enhancer regions downstream FOXP2 and upstream MDFIC. A. Genomic location of human FOXP2 and MDFIC genes. Genes are depicted in blue. B. Detailed view of an Encode UCSC genome-browser snapshot showing bar graphs with a detailed representation of the locations of H3K4Me1 and H3K27Ac histone marks in human neurological cell lines. The squared regions in red show the locations of FOXP2-E $\mathrm{E}^{\text {proximal }}$ and FOXP2-E $\mathrm{E}^{\text {distal }}$. The red asterisk shows the position of the 7q breakpoint in the proband harbouring a genomic complex rearrangement and with severe expressive and receptive speech and language impairment. C. Schematic representation of the location of the four $\operatorname{sgRNAs}$ flanking the $6.2 \mathrm{~kb}$ region including FOXP2- $\mathrm{E}^{\text {proximal }}$ and the $2.3 \mathrm{~kb}$ region including FOXP2-E $\mathrm{E}^{\text {distal }}$.

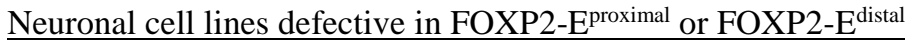




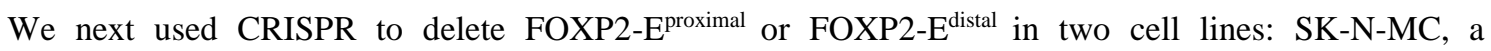
neuroblastomic cell line derived from the supra-orbital area, which expresses FOXP2 constitutively (although it does not express MDFIC at the same level), and SH-SY5Y, a neuroblastomic cell line which expresses neither FOXP2 nor MDFIC. The SH-SY5Y cell line was used as a negative control to confirm that changes produced by CRISPR-Cas9 were not due to unspecific effects. Cells were electroporated with 2ug of either pLV-U6 ${ }^{\# 1} \mathrm{H} 1^{\# 2}-\mathrm{C} 9 \mathrm{G}-\mathrm{E}^{\text {proximal }}, \mathrm{pLV}-\mathrm{U} 6^{\# 1} \mathrm{H} 1^{\# 2}-\mathrm{C} 9 \mathrm{G}-\mathrm{E}^{\text {distal }}$, or with empty plasmids. After $72 \mathrm{~h}$ the DNA was extracted and analysed. PCR and Sanger sequencing analyses confirmed the deletion of the $6.2 \mathrm{~kb}$ or the $2.3 \mathrm{~kb}$ fragment (Figures $2 \mathrm{~A}$ and $2 \mathrm{~B}$ ). We then generated two clonal cell lines (one for each putative enhancer) by sorting GFP positive cells into 96-well plates for single cell colony expansion. We confirmed by PCR that each expanded cellular clone harbours a deletion containing either the FOXP2$E^{\text {proximal }}$ or the FOXP2-E $E^{\text {distal }}$ regions (data not shown). These two cell lines were used for further expression analyses.

\section{$\underline{F O X P 2 \text { and } M D F I C \text { expression analyses }}$}

We next aimed to characterize in more detail the functionality of FOXP2-E proximal $^{\text {and FOXP2-E }}$ distal . We used RT-qPCR to determine the amount of FOXP2 mRNA in the SK-N-MC cells transduced with either pLV-U6 ${ }^{\# 1} \mathrm{H} 1^{\# 2}-\mathrm{C} 9 \mathrm{G}-\mathrm{E}^{\text {proximal }}$, or $\mathrm{pLV}-\mathrm{U} 6^{\# 1} \mathrm{H} 1^{\# 2}-\mathrm{C} 9 \mathrm{G}-\mathrm{E}^{\text {distal }}$, or an empty plasmid as a control. The expression of FOXP2 was significantly reduced (2.9 fold change) compared to that of the control when FOXP2-E proximal was deleted (Figure 2C, left). Likewise, FOXP2 expression was decreased (2 fold change) when FOXP2-E ${ }^{\text {distal }}$ was deleted (Figure 2C left). We then measured the levels of expression of MDFIC after deletion of each enhancer. As shown in Figure 2C right, the expression of MDFIC was significantly increased when either FOXP2-E $\mathrm{E}^{\text {proximal }}$ or FOXP2-E ${ }^{\text {distal }}$ were deleted (8.6 and 7.5 fold change, respectively). The experiment was replicated in SH-SY5Y cells, but no change of expression of FOXP2 or MDFIC was detected (data not shown).

We next analysed by Western blot the amount of FOXP2 and MDFIC proteins in the SK-N-MC cell clones harbouring the FOXP2-E $\mathrm{E}^{\text {proximal }}$ or FOXP2-E $\mathrm{E}^{\text {distal }}$ deletions or control cells transduced with an empty plasmid (Figure 2D). In line with our mRNA data, the deletion of FOXP2-E $E^{\text {proximal }}$ or FOXP2-E ${ }^{\text {distal }}$ was found to reduce the level of FOXP2 (Figure 2D top) and to increase the level of MDFIC (Figure 2D bottom). 
A

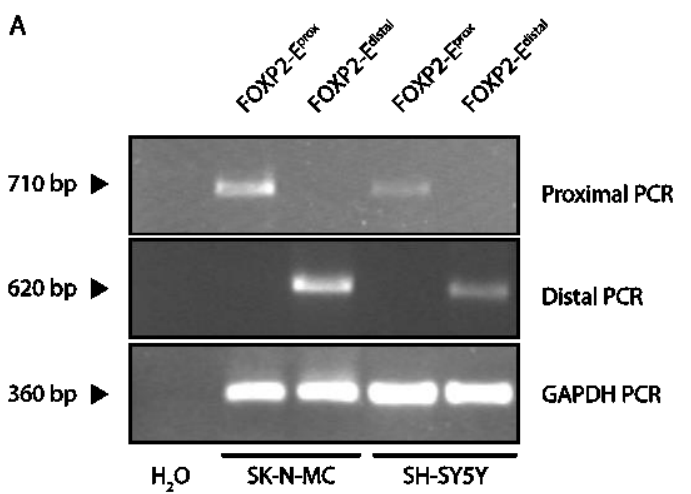

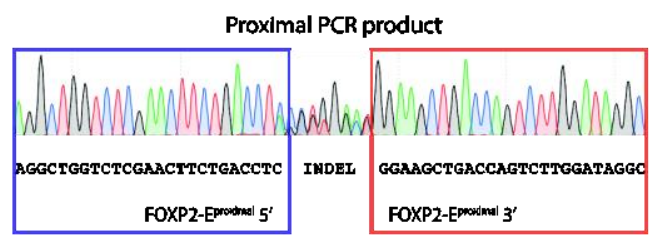

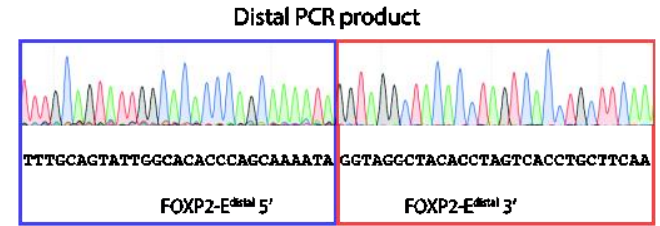

C

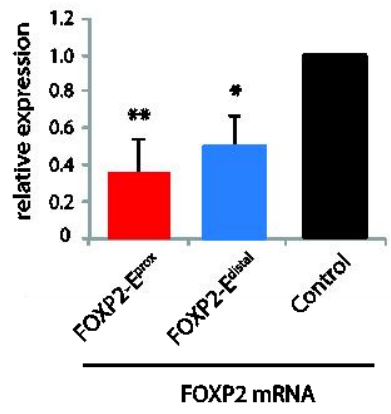

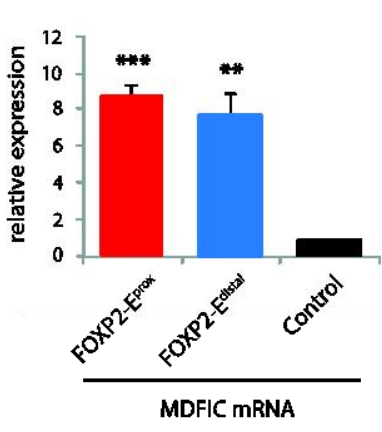

D

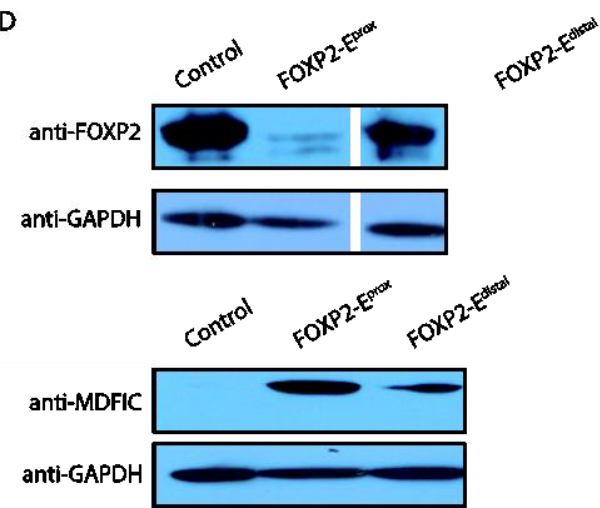

Figure 2. Molecular characterization of FOXP2-E ${ }^{\text {proximal }}$ and FOXP2-E ${ }^{\text {distal }}$. A. PCR analysis. Two oligos flanking the deleted regions were used to amplify the genomic DNA from mutant SK-N-MC and SH-SY-5Y clones. GAPDH genomic oligos were used to amplify a positive control region. Black triangles show the size of the PCR products. B. Representative Sanger sequencing chromatogram showing the

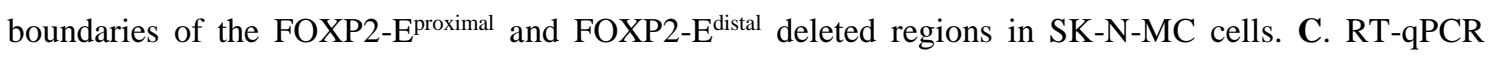
analysis of SK-N-MC cells with FOXP2-E $\mathrm{E}^{\text {proximal }}$ or FOXP2-E $\mathrm{E}^{\text {distal }}$ deletions. Samples are normalized to the average FOXP2 (left) or MDFIC (right) signal between three SK-N-MC wild type replicates. Levels of expression of FOXP2 and MDFIC are represented by the fold change relative to that of parental control cell line, which were normalized to 1 . D. Western blot analysis of cell lysates of SK-N-MC with FOXP2$E^{\text {proximal }}$ deleted, with FOXP2-E $E^{\text {distal }}$ deleted, and of control cells immunoblotted for FOXP2 (top) or MDFIC (bottom).

\section{DISCUSSION}

In this paper we have characterised in detail the role of two functional regulatory elements located downstream FOXP2. One of these enhancers, FOXP2-E ${ }^{\text {distal }}$, had been previously found to be functional in 
a luciferase assay (Becker et al. 2015). We have been able to prove that if deleted, FOXP2 becomes downregulated and the levels of FOXP2 protein are reduced in the SK-N-MC neuroblastomic cell-line. We have further proved that it also affects the adjacent gene, MDFIC. Its deletion upregulates $M D F I C$ and increases the levels of MDFIC protein. The second enhancer, FOXP2-E ${ }^{\text {proximal }}$ was previously unknown. We have found that its deletion also downregulates FOXP2 and upregulates MDFIC. These findings are coherent with previous studies reporting pairs of genes being governed by the same regulatory regions (Gould et al. 1997; Tsujimura et al. 2010), which in some cases have proven to regulate recruitment of RNA polymerase II to promoters of both genes (Collins et al. 2012).

FOXP2 is a well-known gene, important for speech and language (Fisher and Scharff 2009, Graham and Fisher 2013). Less is known about the role of MDFIC in cognitive development and disease. There is evidence that the gene might be associated to language development and cognitive impairment (DECIPHER patients 262086, 292652, and 301696). MDFIC is a MyoD family inhibitor domain containing protein that acts as an activator or repressor of transcription (Thebault et al. 2000, Gautier et al. 2005). Similarly to FOXP2, it interacts with LEF1, as part of beta-catenin regulation (Kusano et al. 2002). In chicken MDFIC is targeted by miR-130a (Han et al. 2016), known to regulate neurite outgrowth and dendritic spine density by targeting $M e C P 2$, the main candidate for Rett syndrome, a condition involving language deficits (Zhang et al. 2016). MDFIC is highly expressed in the cerebellum during human embryonic development and in the thalamus after birth (Human Brain Transcriptome http://hbatlas.org/). These two brain regions, interacting in a dopaminergic cortico-striato-thalamic loop, seem to play an important role in timing sensorimotor control, needed for auditory-motor language processing (Alcock et al. 2000, Vargha-Khadem et al. 2005). The cerebellum and thalamus of those bearing the R553H mutation in $F O X P 2$, associated to speech and language impairment, exhibit volumetric changes of their grey matter (Watkins et al. 2002 a). This suggests that the modulation of the size of neural populations in particular regions in the loop may impact in sensorimotor performance.

Our findings in a human neuronal cell line give support to the view that the breakpoint in our proband, which separated each of these two intergenic enhancers from each other and consequently from one of the two genes they regulate, may have altered the expression levels of both FOXP2 and MDFIC contributing to the observed speech and language deficits (Moralli et al 2015). Whereas MDFIC remained in 
chromosome 7q with, predictably, FOXP2-E distal, FOXP2 was rearranged to chromosome $11 \mathrm{p}$ with,

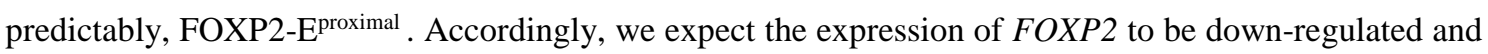
the expression of MDFIC to be up-regulated. The knockdown of FoxP2 in zebra finch results in a shorter window for song learning and in less accurate and shorter song imitation (Haesler et al., 2007). This is coherent with the inability that those carrying the $\mathrm{R} 553 \mathrm{H}$ mutation of $F O X P 2$ show in repeating words and pseudowords (Watkins et al., 2002b). Further confirmation of our hypothesis would need to be supported in a specific neuronal brain cell line grown from stem cells of the proband, as well as in songbirds in which these enhancers have been deleted.

We expect that our findings also contribute to a better understanding of the role that this region may have played in the evolution of language. Differences in the expression levels of both FOXP2 and MDFIC are expected between extinct hominins and modern humans, plausibly accounting for some of the presumed differences in their language abilities. Neanderthals bear the ancestral allele of a binding site for POU3F2 within intron 8 of FOXP2, which is more efficient in activating transcription (Maricic et al. 2013). Accordingly, higher levels of FOXP2 are expected in this hominin species. Likewise, the MDFIC locus is among the top five percent S score regions in modern humans (Green et al. 2010, table S37). Finally, both genes are functionally related to $R U N X 2$, which encodes an osteogenic factor that controls the closure of cranial sutures and several aspects of brain growth, and that has been related to the changes that brought about our more globular brain (case) and our species-specific mode of cognition, including language (Boeckx and Benítez-Burraco 2014; Benítez-Burraco and Boeckx 2015). Further confirmation of this hypothesis would need to be supported by the analysis of the enhancers' sequences in extinct hominins and by mimicking the attested changes (if any) in a human cell line.

Ii is expected that our study, together with new available data about seed sequences of miRs in the 3'UTR region of FOXP2 (Clovis et al. 2012; Shi et al. 2013; Fu et al. 2014a; Cuiffo et al. 2014), contributes to a deeper understanding of how FOXP2 is regulated. Since we have found that both adjacent genes, FOXP2 and $M D F I C$, are sharing a regulatory region downstream the 3 ' end of FOXP2 which modulates their expression, it is expected that these results will contribute as well to a better understanding of how each gene participates in the development of subcellular and sensorimotor neural function underlying language. 


\section{REFERENCES}

Alcock KJ, Passingham RE, Watkins KE, Vargha-Khadem F. (2000) Pitch and timing abilities in inherited speech and language impairment. Brain Lang 75 (1): 34-46. doi: 10.1006/brin.2000.2323

Becker M, Devanna P, Fisher SE, Vernes SC (2015) A chromosomal rearrangement in a child with severe speech and language disorder separates FOXP2 from a functional enhancer. Mol Cytogenet 8:69. doi: 10.1186/s13039-015-0173-0

Bejerano, G., et al., 2004. Ultraconserved elements in the human genome. Science 304: 1321-1325. doi: 10.1126/science.1098119

Benítez-Burraco A, Boeckx C (2015) Approaching motor and language deficits in autism from below: a biolinguistic perspective. Front Integr Neurosci 9:25. doi: 10.3389/fnint.2015.00025

Boeckx C, Benítez-Burraco A (2014) The shape of the human language-ready brain. Front Psychol 5:282. doi: 10.3389/fpsyg.2014.00282

Bonkowsky JL, Wang X, Fujimoto E, et al. (2008) Domain-specific regulation of foxP2 CNS expression by lef1. BMC Dev Biol 8:103. doi: 10.1186/1471-213X-8-103

Bruce HA, Margolis RL (2002) FOXP2: novel exons, splice variants, and CAG repeat length stability. Hum Genet 111:136-144. doi: 10.1007/s00439-002-0768-5

Chandrasekaran B, Yi H-G, Blanco NJ, et al. (2015) Enhanced procedural learning of speech sound categories in a genetic variant of FOXP2. J Neurosci 35:7808-7812. doi: 10.1523/JNEUROSCI.470614.2015

Chiu Y-C, Li M-Y, Liu Y-H, et al. (2014) Foxp2 regulates neuronal differentiation and neuronal subtype specification. Dev Neurobiol 74:723-738. doi: 10.1002/dneu.22166

Clovis YM, Enard W, Marinaro F, et al. (2012) Convergent repression of Foxp2 3'UTR by miR-9 and miR132 in embryonic mouse neocortex: implications for radial migration of neurons. Development 139:3332-3342. doi: 10.1242/dev.078063

Collins PL, Henderson MA, Aune TM. (2012) Lineage-specific adjacent IFNG and IL26 genes share a common distal enhancer element. Genes Immun. 13:481-488. doi: 10.1038/gene.2012.22.

Cong L, Ran FA, Cox D, et al. (2013) Multiplex genome engineering using CRISPR/Cas systems. Science 339:819-823. doi: 10.1126/science.1231143

Cuiffo BG, Campagne A, Bell GW, et al. (2014) MSC-regulated microRNAs converge on the transcription factor FOXP2 and promote breast cancer metastasis. Cell Stem Cell 15:762-774. doi: 10.1016/j.stem.2014.10.001

Fisher SE, Scharff C (2009) FOXP2 as a molecular window into speech and language. Trends Genet 25:166-177. doi: 10.1016/j.tig.2009.03.002

Fu Y, Sander JD, Reyon D, et al. (2014a) Improving CRISPR-Cas nuclease specificity using truncated guide RNAs. Nat Biotechnol 32:279-284. doi: 10.1038/nbt.2808

Fu L, Shi Z, Luo G, et al. (2014b) Multiple microRNAs regulate human FOXP2 gene expression by targeting sequences in its 3' untranslated region. Mol Brain 7:71. doi: 10.1186/s13041-014-0071-0

Garcia-Calero E, Botella-Lopez A, Bahamonde O, et al. (2016) FoxP2 protein levels regulate cell morphology changes and migration patterns in the vertebrate developing telencephalon. Brain Struct Funct 221:2905-2917. doi: 10.1007/s00429-015-1079-7

Gautier VW, Sheehy N, Duffy M., et al. (2005) Direct interaction of the Human I-mfa domain- containing 
protein, HIC, with HIV-1 TAT results in cytoplasmic sequestration and control of Tat activity. Proc Natl Acad Sci USA 102:16362-16367

Gould A, Morrison A, Sproat G, et al. (1997) Positive cross-regulation and enhancer sharing: two mechanisms for specifying overlapping Hox expression patterns. Genes Dev. 11:900-913. doi 10.1101/gad.11.7.900. doi: 10.1073/pnas.0503519102

Graham SA, Fisher SE (2013) Decoding the genetics of speech and language. Curr Opin Neurobiol 23:4351. doi: 10.1016/j.conb.2012.11.006

Green RE, Krause J, Briggs AW, et al. (2010) A draft sequence of the Neandertal genome. Science 328:710-722. doi: 10.1126/science. 1188021

Haesler S, Rochefort C, Georgi B, et al. (2007) Incomplete and inaccurate vocal imitation after knockdown of FoxP2 in songbird basal ganglia nucleus area X. PLoS Biol. 5:e321. doi: 10.1371/journal.pbio.0050321

Hallikas O, Palin K, Sinjushina N, Rautiainen R, Partanen J, Ukkonen E, Taipale J: Genome-wide prediction of mammalian enhancers based on analysis of transcription-factor binding affinity. Cell. 2006, 124: 47-59.

Han B, Lian L, Li X, et al. (2016) Chicken gga-miR-130a targets HOXA3 and MDFIC and inhibits Marek's disease lymphoma cell proliferation and migration. Mol Biol Rep 43:667-76. doi: 10.1007/s11033016-4002-2.

Hoogman M, Guadalupe T, Zwiers MP, et al. (2014) Assessing the effects of common variation in the FOXP2 gene on human brain structure. Front Hum Neurosci 8:473. doi: 10.3389/fnhum.2014.00473

Kurt S, Fisher SE, Ehret G (2012) Foxp2 mutations impair auditory-motor association learning. PLoS ONE 7:e33130. doi: 10.1371/journal.pone.0033130

Kusano S, Raab-Traub N. (2002) I-mfa domain proteins interact with Axin and affect its regulation of the Wnt and c-Jun N-terminal kinase signaling pathways. Mol Cell Biol 22:6393-405. doi: 10.1128/MCB.22.18.6393-6405.2002

Lai CS, Gerrelli D, Monaco AP, et al. (2003) FOXP2 expression during brain development coincides with adult sites of pathology in a severe speech and language disorder. Brain 126:2455-62. doi: http://dx.doi.org/10.1093/brain/awg247 2455-2462

Mali P, Yang L, Esvelt KM, et al. (2013) RNA-guided human genome engineering via Cas9. Science 339:823-826. doi: 10.1126/science.1232033

Maricic T, Günther V, Georgiev O, et al. (2013) A recent evolutionary change affects a regulatory element in the human FOXP2 gene. Mol Biol Evol 30:844-852. doi: 10.1093/molbev/mss271

Moralli D, Nudel R, Chan MTM, et al. (2015) Language impairment in a case of a complex chromosomal rearrangement with a breakpoint downstream of FOXP2. Mol Cytogenet 8:36. doi: 10.1186/s13039015-0148-1

Mueller KL, Murray JC, Michaelson JJ, et al. (2016) Common genetic variants in FOXP2 are not associated with individual differences in language development. PLoS ONE 11:e0152576. doi: 10.1371/journal.pone.0152576

Padovani A, Cosseddu M, Premi E, et al. (2010) The speech and language FOXP2 gene modulates the phenotype of frontotemporal lobar degeneration. J Alzheimers Dis 22:923-931. doi: 10.3233/JAD2010-101206

Rodriguez-Perales S, Torres-Ruiz R, Suela J, et al. (2015) Truncated RUNX1 protein generated by a novel $\mathrm{t}(1 ; 21)(\mathrm{p} 32 ; \mathrm{q} 22)$ chromosomal translocation impairs the proliferation and differentiation of human 
hematopoietic progenitors. Oncogene. doi: 10.1038/onc.2015.70

Sakuma T, Woltjen K (2014) Nuclease-mediated genome editing: At the front-line of functional genomics technology. Dev Growth Differ 56:2-13. doi: 10.1111/dgd.12111

Schroeder DI, Myers RM (2008) Multiple transcription start sites for FOXP2 with varying cellular specificities. Gene 413:42-48. doi: 10.1016/j.gene.2008.01.015

Shi Z, Luo G, Fu L, et al. (2013) miR-9 and miR-140-5p target FoxP2 and are regulated as a function of the social context of singing behavior in zebra finches. J Neurosci 33:16510-16521. doi: 10.1523/JNEUROSCI.0838-13.2013

Thebault, S., Gachon, F., Lemasson, I., et al. (2000) Molecular cloning of a novel human I-mfa domaincontaining protein that differently regulates human T-cell leukemia virus type I and HIV-1 expression. J Biol Chem 275: 4848-4857]. doi: 10.1074/jbc.275.7.4848

Tolosa A, Sanjuán J, Dagnall AM, et al. (2010) FOXP2 gene and language impairment in schizophrenia: association and epigenetic studies. BMC Med Genet 11:114. doi: 10.1186/1471-2350-11-114

Torres R, Martin MC, Garcia A, et al. (2014a) Engineering human tumour-associated chromosomal translocations with the RNA-guided CRISPR-Cas9 system. Nature Communications. doi: $10.1038 /$ ncomms4964

Torres R, Garcia A, Jimenez M, et al. (2014b) An integration-defective lentivirus-based resource for sitespecific targeting of an edited safe-harbour locus in the human genome. Gene Ther 21:343-352. doi: 10.1038/gt.2014.1

Torres-Ruiz R, Rodriguez-Perales S (2016) CRISPR-Cas9 technology: applications and human disease modelling. Brief Funct Genomics elw025. doi: 10.1093/bfgp/elw025

Tsui D, Vessey JP, Tomita H, et al. (2013) FoxP2 regulates neurogenesis during embryonic cortical development. J Neurosci 33:244-258. doi: 10.1523/JNEUROSCI.1665-12.2013

Tsujimura Tl, HosoyaT, Kawamara S (2010) A single enhancer regulating the differential expression of duplicated red-sensitive opsine genes in zebra-fish. PLoS Genet. 2010 Dec 16;6 (12):e1001245. doi:10.1371/journal.pgen.1001245

Vargha-Khadem F, Gadian DG, Copp A, Mishkin M (2005) FOXP2 and the neuroanatomy of speech and language. Nat Rev Neurosci 6:131-138. doi: 10.1038/nrn1605.

Watkins KE, Vargha-Khadem F, Ashburner J, et al. (2002a) MRI analysis of an inherited speech and language disorder: structural brain abnormalities. Brain 125:465-478.

Watkins KE, Dronkers NF, Vargha-Khadem F (2002b) Behavioural analysis of an inherited speech and language disorder: comparison with acquired aphasia. Brain 125:452-464.

Zhang Y, Chen M, Qiu Z, et al. (2016) MiR-130a regulates neurite outgrowth and dendritic spine density by targeting MeCP2. Protein Cell 7:489-500. doi: 10.1007/s13238-016-0272-7.

Zhao Y, Ma H, Wang Y, et al. (2010) Association between FOXP2 gene and speech sound disorder in Chinese population. Psychiatry Clin Neurosci 64:565-573. doi: 10.1111/j.1440-1819.2010.02123.x

Supplementary Table 1. Oligonucleotide and sgRNA sequences used in this study. 


\section{sgRNA sequences (IDT gblocks) sgRNA_FOXP2_Edistal_1_5:}

ccataACGCGTTGTACACGAACGCTGACGTCATCAACCCGCTCCAAGGAATCGCGGGCC CAGTGTCACTAGGCGGGAACACCCAGCGCGCGTGCGCCCTGGCAGGAAGATGGCTGTGA GGGACAGGGGAGTGGCGCCCTGCAATATTTGCATGTCGCTATGTGTTCTGGGAAATCAC CATAAACGTGAAATGTCTTTGGATTTGGGAATCTTATAAGTTCTGTATGAGACCACTCT TTCCCGcacacccagcaaatacatGTTTTAGAGCTATGCTGGAAACAGCATAGCAAGT TAAAATAAGGCTAGTCCGTTATCAACTTGAAAAAGTGGCACCGAGTCGGTGCTTTTTTA CTAGTcgeta

\section{sgRNA_FOXP2_Edistal_2_5:}

ccataACGCGTTGTACACGAACGCTGACGTCATCAACCCGCTCCAAGGAATCGCGGGCC CAGTGTCACTAGGCGGGAACACCCAGCGCGCGTGCGCCCTGGCAGGAAGATGGCTGTGA GGGACAGGGGAGTGGCGCCCTGCAATATTTGCATGTCGCTATGTGTTCTGGGAAATCAC CATAAACGTGAAATGTCTTTGGATTTGGGAATCTTATAAGTTCTGTATGAGACCACTCT TTCCCGgcaaggtatattctctgagGTTTTAGAGCTATGCTGGAAACAGCATAGCAAGT TAAAATAAGGCTAGTCCGTTATCAACTTGAAAAAGTGGCACCGAGTCGGTGCTTTTTTA CTAGTcgeta

\section{sgRNA_FOXP2_Edistal_1_3:}

ccataCAATTGGGGCAGGAAGAGGGCCTATTTCCCATGATTCCTTCATATTTGCATATA CGATACAAGGCTGTTAGAGAGATAATTAGAATTAATTTGACTGTAAACACAAAGATATT AGTACAAAATACGTGACGTAGAAAGTAATAATTTCTTGGGTAGTTTGCAGTTTTAAAAT TATGTTTTAAAATGGACTATCATGTACACTTACCGTAACTTGAAAGTATTTCGATTTCT TGGCTTTATATATCTTGTGGAAAGGACGAGGTACCGatctactcttctttagggtGTTT TAGAGCTATGCTGGAAACAGCATAGCAAGTTAAAATAAGGCTAGTCCGTTATCAACTTG AAAAAGTGGCACCGAGTCGGTGCTTTTTTACGCGTACTAGTcgcta

\section{sgRNA_FOXP2_Edistal_2_3:}

ccataCAATTGGGGCAGGAAGAGGGCCTATTTCCCATGATTCCTTCATATTTGCATATA CGATACAAGGCTGTTAGAGAGATAATTAGAATTAATTTGACTGTAAACACAAAGATATT AGTACAAAATACGTGACGTAGAAAGTAATAATTTCTTGGGTAGTTTGCAGTTTTAAAAT TATGTTTTAAAATGGACTATCATGTACACTTACCGTAACTTGAAAGTATTTCGATTTCT TGGCTTTATATATCTTGTGGAAAGGACGAGGTACCGgaagagtagatcgcatgagGTTT TAGAGCTATGCTGGAAACAGCATAGCAAGTTAAAATAAGGCTAGTCCGTTATCAACTTG AAAAAGTGGCACCGAGTCGGTGCTTTTTTACGCGTACTAGTcgeta 


\section{sgRNA_FOXP2_Eproximal_1_5:}

ccataACGCGTTGTACACGAACGCTGACGTCATCAACCCGCTCCAAGGAATCGCGGGCC CAGTGTCACTAGGCGGGAACACCCAGCGCGCGTGCGCCCTGGCAGGAAGATGGCTGTGA GGGACAGGGGAGTGGCGCCCTGCAATATTTGCATGTCGCTATGTGTTCTGGGAAATCAC CATAAACGTGAAATGTCTTTGGATTTGGGAATCTTATAAGTTCTGTATGAGACCACTCT TTCCCGgtgatctcagctactcgggGTTTTAGAGCTATGCTGGAAACAGCATAGCAAG TTAAAATAAGGCTAGTCCGTTATCAACTTGAAAAAGTGGCACCGAGTCGGTGCTTTTTT ACTAGTcgeta

\section{sgRNA_FOXP2_Eproximal_2_5:}

ccataACGCGTTGTACACGAACGCTGACGTCATCAACCCGCTCCAAGGAATCGCGGGCC CAGTGTCACTAGGCGGGAACACCCAGCGCGCGTGCGCCCTGGCAGGAAGATGGCTGTGA GGGACAGGGGAGTGGCGCCCTGCAATATTTGCATGTCGCTATGTGTTCTGGGAAATCAC CATAAACGTGAAATGTCTTTGGATTTGGGAATCTTATAAGTTCTGTATGAGACCACTCT TTCCCGctcgaacttctgacctcagGTTTTAGAGCTATGCTGGAAACAGCATAGCAAGT TAAAATAAGGCTAGTCCGTTATCAACTTGAAAAAGTGGCACCGAGTCGGTGCTTTTTTA CTAGTcgeta

\section{sgRNA_FOXP2_Eproximal_1_3:}

ccataCAATTGGGGCAGGAAGAGGGCCTATTTCCCATGATTCCTTCATATTTGCATATA CGATACAAGGCTGTTAGAGAGATAATTAGAATTAATTTGACTGTAAACACAAAGATATT AGTACAAAATACGTGACGTAGAAAGTAATAATTTCTTGGGTAGTTTGCAGTTTTAAAAT TATGTTTTAAAATGGACTATCATGTACACTTACCGTAACTTGAAAGTATTTCGATTTCT TGGCTTTATATATCTTGTGGAAAGGACGAGGTACCGctgtaataagatagcagggGTTT TAGAGCTATGCTGGAAACAGCATAGCAAGTTAAAATAAGGCTAGTCCGTTATCAACTTG AAAAAGTGGCACCGAGTCGGTGCTTTTTTACGCGTACTAGTcgcta

\section{sgRNA_FOXP2_Eproximal_2_3:}

ccataCAATTGGGGCAGGAAGAGGGCCTATTTCCCATGATTCCTTCATATTTGCATATA CGATACAAGGCTGTTAGAGAGATAATTAGAATTAATTTGACTGTAAACACAAAGATATT AGTACAAAATACGTGACGTAGAAAGTAATAATTTCTTGGGTAGTTTGCAGTTTTAAAAT TATGTTTTAAAATGGACTATCATGTACACTTACCGTAACTTGAAAGTATTTCGATTTCT TGGCTTTATATATCTTGTGGAAAGGACGAGGTACCGtatggctgccacattccgtGTTT TAGAGCTATGCTGGAAACAGCATAGCAAGTTAAAATAAGGCTAGTCCGTTATCAACTTG AAAAAGTGGCACCGAGTCGGTGCTTTTTTACGCGTACTAGTcgcta 


\title{
Primers qPCR
}

$>$ qFOXP2_FW

GCAGCAGAGATGGAAGATCA

$>$ qFOxP2_Rv

AGTTGTCTTGCTGCCTGGAG

cDNA amplicon size:103

Estimated genomic amplicon size:108040

$>$ QMDFIC_FW

GTCAATTTGGGGAAATCCTT

$>$ qMDFIC RV

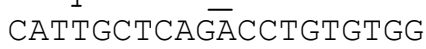

CDNA amplicon size:140

Estimated genomic amplicon size:37248

\section{Primers Surveyor \& deletion detection}

SMDFIC Fw2

TGATCTCAGTGCAGGCAAA

SMDFIC RV2

GTTGGACTAAGGTGCCAGTT

2314pb (deletion FOXP2 Distal)

\author{
SMDFIC FW \\ TACTGTTTCATGGATGCTGACT \\ SMDFIC RV \\ CCTTTGGCCACAGACTGAA
}

\section{SFOXP2 FW}

GGGATAGCACTGGGAGAAATAC

SFOXP2 RV

GCGGTGGCTCATTTCTGTA

6264 pb (deletion FOXP2 Proximal)

SFOXP2 FW2

TTCTGCACCTTGGGTTAGG

SFOXP2 Rv2

AGGGTTGATTGATTGCCAGAG 